\title{
NoteCoStruct: Powering Online Learners with Socially Scaffolded Note Taking and Sharing
}

\author{
Jingchao Fang \\ jcfang@ucdavis.edu \\ University of California, Davis \\ Davis, California, USA \\ Chi-Lan Yang \\ chilan.yang@cyber.t.u-tokyo.ac.jp \\ The University of Tokyo \\ Tokyo, Japan
}

\author{
Yanhao Wang \\ wangyanhao@zju.edu.cn \\ Zhejiang University \\ Zhejiang, China \\ Hao-Chuan Wang \\ hciwang@ucdavis.edu \\ University of California, Davis \\ Davis, California, USA
}

\begin{abstract}
Video-based learning and digital note-taking are widely adopted by online learners. To investigate how to better support self-paced online learners' note-taking and learning activities, we presented and evaluated NoteCoStruct, a digital note-taking and note-sharing tool for video-based learning by scaffolding the learners through a structured note-taking process and displaying learning traces left by peers watching the same videos before. With an online laboratory study involving 20 participants, we found that NoteCoStruct significantly reduced perceived distraction caused by note-taking, fostered a sense of learning community, and better engaged learners cognitively and emotionally in video-based learning. We discussed our findings and outlined possible future work around digital notetaking and note-sharing tools.
\end{abstract}

\section{CCS CONCEPTS}

- Human-centered computing $\rightarrow$ Collaborative and social computing systems and tools.

\section{KEYWORDS}

Video-based learning; Online learning; Digital note-taking; Sense of community

\section{ACM Reference Format:}

Jingchao Fang, Yanhao Wang, Chi-Lan Yang, and Hao-Chuan Wang. 2021. NoteCoStruct: Powering Online Learners with Socially Scaffolded Note Taking and Sharing. In CHI Conference on Human Factors in Computing Systems Extended Abstracts (CHI '21 Extended Abstracts), May 8-13, 2021, Yokohama, Japan. ACM, New York, NY, USA, 5 pages. https://doi.org/10. $1145 / 3411763.3451694$

\section{INTRODUCTION}

Video-based learning has become widely employed in the past decade and it has many advantages over the traditional model of teaching and learning. Students are able to learn by watching

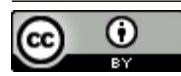

This work is licensed under a Creative Commons Attribution International 4.0 License.

CHI '21 Extended Abstracts, May 8-13, 2021, Yokohama, Japan

(c) 2021 Copyright held by the owner/author(s).

ACM ISBN 978-1-4503-8095-9/21/05.

https://doi.org/10.1145/3411763.3451694 pre-recorded lectures anytime and anywhere; nevertheless, such asynchronous and remote nature of video-based learning also introduces challenges. For instance, students face difficulties in interacting with other learners in the same class and risk negative impacts due to isolation [1]. Also, their needs of exchanging and sharing thoughts and ideas with peer learners can hardly be supported as in traditional classrooms [20].

As a cohort with great commonality (e.g., shared academic interests, or common goal of passing the class), online learners of the same class can be seen as a learning community [4,21]. Considering the two elements of sense of community are group membership (i.e., feeling of belonging to a group of people) and needs fulfillment (i.e., individuals' needs can be met by the community) [21], whether learners perceive a sense of learning community may have a significant impact on their learning experience. Enhancing online learners' sense of community can be an effective way to mitigate some of the challenges raised by asynchronicity and distance in online learning, while lack of it could have negative effects such as greater course dissatisfaction and lower retention rate $[2,10]$.

Efforts have been made to foster online learners' sense of community. For instance, it's a common design pattern for learning platforms to feature discussion forums, which are shown to be useful for enhancing the visibility of peer learners and sense of social co-presence [16]. However, in practice, only a small portion of learners participate in such forums [3]. Students may be reluctant to actively participate in discussion forums as asynchronous postings may appear to be slow and not energetic [9]. Other designs have been looking into time-anchored comments left by previous learners when encountering the same piece of content (e.g., specific playback time in a video), and found that dynamically displaying time-anchored comments in educational videos can effectively enhance learners' perceived social interactivity, and content of displayed comments may influence subsequent learners' intention of contributing their comments [14].

We identified the opportunity for self-paced learners to share independently created notes during their individual courses of learning with subsequent learners to foster a sense of learning community, and to provide extra social scaffold for video-based learning. Here, we define digital notes as condensed records and/or brief reflections of the course content. Since note-taking is widely adopted by online learners and naturally embedded in the learning process, 
taking notes for sharing does not introduce extra efforts to individual learners other than the learning activity itself. Thus, they may be motivated to contribute to the community more actively through note-sharing than through forum posting or time-anchored commenting approach. As an external artifact created by learners [11], notes may potentially include learners' specific thoughts on course content, which could be meaningful and valuable information to share with the rest of the learning community. Such information may include worked-out examples and partially processed knowledge that can provide hints of comprehension and facilitate peer learning. From the angle of learners exposed to others' notes, the shared notes can be seen as a form of peers' digital learning traces, which can provide them social contexts to enhance the visibility of peers' learning behaviors and a sense of learning in a community [18], and may further elevate their level of engagement while consuming video lectures [13].

Yet, note-taking remains a challenging task. One major difficulty learners face is that taking full notes while watching video lectures is cognitively demanding and distracting, as note-taking costs extra attention, memory and reasoning efforts for handling rapid and dense lecture presentations $[8,15]$. Though being able to access the transcripts of video recordings may bolster note-taking heuristics like copy-and-pastes, studies showed that directly transcribing lectures verbatim as notes requires shallow cognitive processing and may actually lead to poor learning outcomes [7, 17, 22].

The potential benefits of note-sharing on enhancing sense of community and facilitating peer learning along with the challenges of digital note-taking motivated us to design a system to improve the note-taking and learning experience of online learners from two perspectives. (1) We aim to provide cognitive support for notetaking and help reduce learners' cognitive load of taking notes while learning with videos. (2) We intend to provide social scaffold for isolated learners and enhance their sense of learning community and level of engagement by curating learning traces from peers and enabling note-sharing.

We present NoteCoStruct, a social note-taking system which supports structured note-sharing among learners. We are inspired by previous work which attempted to resolve challenges individual note-takers faced by guiding them through a structured process that breaks holistic note-taking activities into several less demanding interconnected phases of tasks, leading to notes with extensive elaborations and interpretations summarized by individual learners rather than verbatim text copy-pasted from the transcript [15]. In NoteCoStruct, we similarly scaffold users' note-taking activities with three undemanding phases (i.e., highlighting, commenting, and note finalizing). Additionally, on the one hand, users can access traces left by previous learners in each phase, and on the other hand, their outcomes of each phase can serve as learning traces which are potentially rich sources for subsequent learners. With structured note-taking and note-sharing, besides reducing learners' cognitive loads when taking notes while watching lecture videos, we also expect that the shared notes generated by learners using NoteCoStruct can be easier to consume because they tend to be lighter than holistic notes.

With a between-subject controlled experiment, we compared NoteCoStruct with the baseline of a holistic note-taking and notesharing system to investigate its effectiveness. Our results showed that participants who learned with NoteCoStruct reported to perceive a higher sense of community, and were more cognitively and emotionally engaged during note-taking and learning. They also felt less distracted due to the structured note-taking process. We discussed the implications and future work for designing social note-taking systems for online learners.

\section{SYSTEM DESIGN}

\subsection{NoteCoStruct}

NoteCoStruct is designed as a structured social note-taking and note-sharing prototype which scaffolds learners' to take digital notes with an undemanding three-phase process and presents peers' learning traces in the forms of highlights, comments and finalized full notes in each phase accordingly.

2.1.1 Phase 1: Video-watching and transcript highlighting. In this first phase, the learners watch the lecture video and are provided with the corresponding time-anchored transcript. Learners are prompted to highlight the parts of the transcript that they think are important. As one of the most widespread learning strategies, highlighting helps students to pay attention to important information [23]. Since highlighting is a lightweight task and requires much less time and effort than writing down full notes, learners can be less distracted from the ongoing lecture. To fully utilize previous learners' learning traces, we visualize and present their highlights by putting a green vertical bar on the left side of the highlighted parts of the transcript, where darker green means highlighted by more learners. The interface of Phase 1 is shown in Figure 1.

2.1.2 Phase 2: Commenting on highlighted parts. One of the major functions of highlighting on learning materials is to transfer the information into working memory for further process [23]. In the second phase, we explicitly ask learners to comment on their previously highlighted parts one by one in order to trigger learners' deeper cognitive processing of the learning material. Instead of merely transcribing the presented information, which is not an effective strategy for learning [17], we encourage learners to provide supporting details, examples, and their own understanding. Extended from Phase 1, we also showed learners the comments written down by previous learners. Since checking out peers' short comments is less demanding than reading their full notes, learners may feel less stressful and more willing to digest such material provided by peers. Figure 2 shows the interface of Phase 2 .

2.1.3 Phase 3: Notes finalization. Preliminary notes are automatically generated based on learners' highlights and comments in the previous two phases (see Figure 3), and it can serve as a starting point for notes finalizing. In the third phase, learners will be able to freely edit the preliminary notes (e.g., adding additional information, deleting unnecessary or redundant parts, or changing the layouts) to generate full notes. Previous peers' full notes are also displayed in this phase for learners' reference.

Altogether, NoteCoStruct employs a structured note-taking process and presents rich learning traces left by learners' peers to provide learning support and foster a sense of community. 


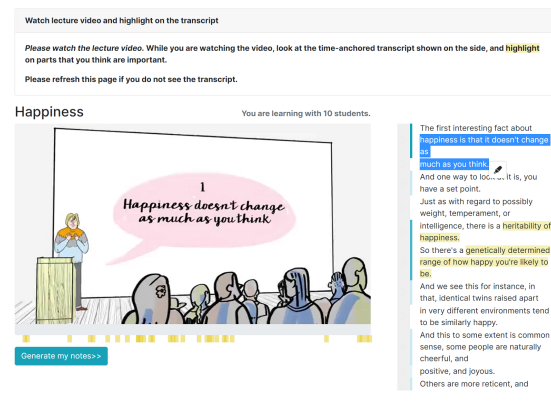

Figure 1: NoteCoStruct Phase 1: Videowatching and transcript highlighting

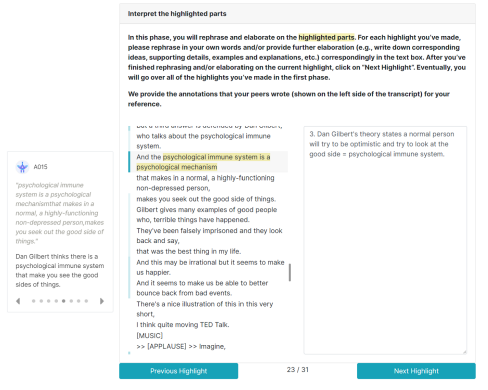

Figure 2: NoteCoStruct Phase 2: Commenting on highlighted parts

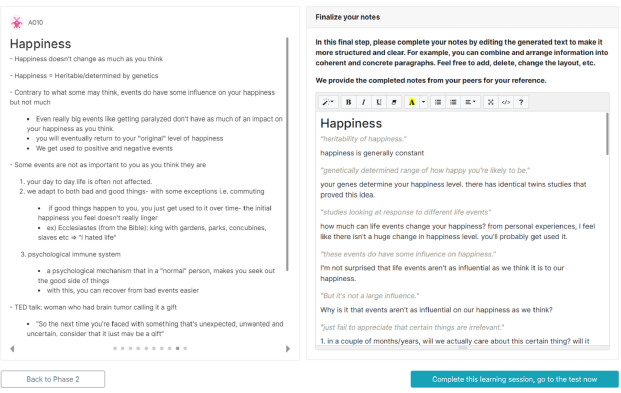

Figure 3: NoteCoStruct Phase 3: Notes finalization

\subsection{Prototyping Social Note-sharing}

To collect learning traces to be shown in NoteCoStruct, we recruited 11 participants to go through two learning sessions and take notes using only the independent note-taking function of NoteCoStruct in which no social cues were shown. One learning session included a 10-minute lecture video and the other included a 4-minute lecture video, with the order of videos counterbalanced. Both videos are retrieved from university-level psychology classes on Coursera ${ }^{1}$, and no prior knowledge is needed to understand the content of lecture videos. We excluded the notes from one participant who did not perform serious learning and note-taking (specifically, did not play the lecture video), thus only social notes from 10 participants were included. In total, participants generated 220 highlights and 198 comments for the 10-minute lecture, 120 highlights and 105

\footnotetext{
${ }^{1}$ https://www.coursera.org
}

comments for the 4-minute lecture, and 10 pieces of full notes for each lecture video.

\section{METHOD}

\subsection{Designing System for Baseline Condition}

To investigate the effectiveness of learning with NoteCoStruct, we designed a baseline system in order to compare our proposed system to it in a controlled online study. The baseline system supports basic video-watching and digital note-taking functions (i.e., holistic notetaking without the three-phase structure) and presents peers' notes (see Figure 4). The note-sharing feature in the baseline system is designed to be comparable to common practices of sharing only peers' full notes without detailed learning traces in the forms of highlights and comments, which is commonplace among students in traditional face-to-face classes. Online discussion forums can technically support this basic note-sharing function as well.

\subsection{Participants and Study Design}

To compare the effect of learning with NoteCoStruct and the baseline system, we recruited a total of 20 participants (12 female, 8 male) and conducted a between-subject experiment. Participants were recruited from an online recruitment platform (Prolific ${ }^{2}$ ), social media (Facebook groups and Reddit) and a mailing list of a public university in the US. All of them are currently undergraduate or graduate students living in the US.

At the beginning of the study, they were given an overview of the process. Participants went through two learning sessions, a test after each session, and a survey at the end of the study. Each learning session either includes the 10-minute video or the 4-minute video provided to the previously recruited 11 note-takers, with the order of videos counterbalanced. They were given a short tutorial of what functions are supported and how to interact with the interface at the beginning of each phase of the learning sessions. Participants were randomly assigned to either learn and take notes with NoteCoStruct $(\mathrm{n}=10)$ or the baseline system $(\mathrm{n}=10)$ in both sessions. Participants took 61.2 minutes on average $(\mathrm{M}=79.4$ minutes with NoteCoStruct, and $M=43.0$ minutes with the baseline system) to complete the task, and each of them received $\$ 10$ compensation.

\subsection{Preliminary Data Analysis and Result}

As a preliminary data analysis, we collected and analyzed participants' responses to survey items which were answered after they experienced two learning sessions with either NoteCoStruct or baseline system. All survey items were presented in 7-point Likert scale.

3.3.1 Cognitive effect to note-taking. To verify whether the scaffolded social note-taking and sharing design caused less distraction from the current learning activity, we analyzed their perceived level of distraction caused by note-taking while watching lecture videos. Two items adopted from [12] (e.g., "I can concentrate in classes when taking notes") were used.

\footnotetext{
${ }^{2}$ https://www.prolific.co/
} 

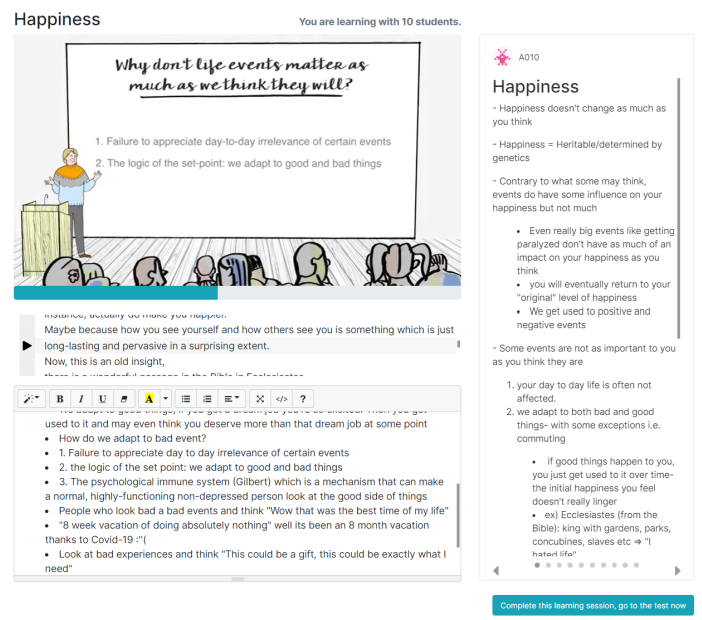

Figure 4: Baseline system supports basic notetaking and note-sharing

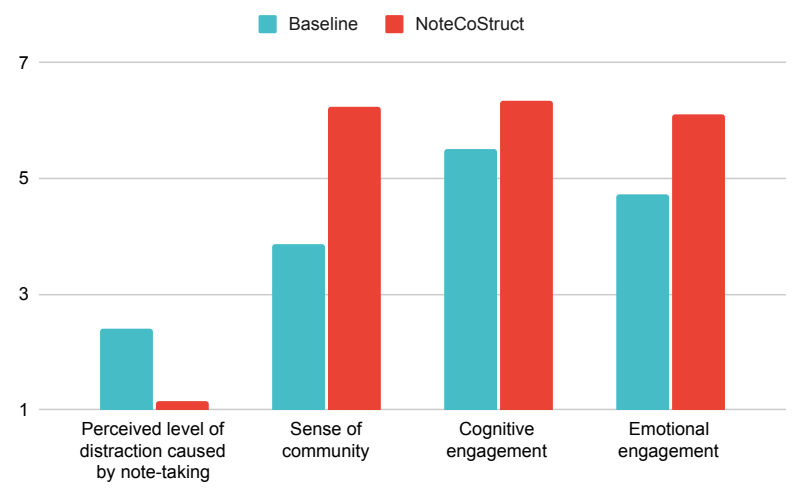

Figure 5: Comparing NoteCoStruct with the baseline system

Results from one-way ANOVA showed that learners reported being significantly less distracted when taking notes with NoteCoStruct, compared to the baseline system $(\mathrm{F}(1,18)=9.75, \mathrm{p}<0.01$. Baseline $(\mathrm{M}=2.40, \mathrm{SD}=1.13)$, NoteCoStruct $(\mathrm{M}=1.15, \mathrm{SD}=0.58))$.

3.3.2 Social scaffolding effects to video-based learning. To assess whether our approach of curating learning traces can enhance learners' sense of community, we used three items adopted from [21] (e.g., "I feel like a member of the community formed by the learners of these two classes") to measure learners' awareness of learning in a community. Additionally, since previous literature suggested that sense of community is closely related to engagement [24], which is an important factor affecting learning outcome [6], we measured cognitive engagement which is critical to the learning from video contents [19] (e.g., "When I am taking the courses with this tool, I am absorbed by course content") and emotional engagement which focuses more on the affective aspects of learning [19] (e.g., "I am enthusiastic about learning online with this tool") using three items for each, all adopted from [5].

One-way ANOVA showed that learners using NoteCoStruct perceived a significantly higher sense of community than using the baseline system $(\mathrm{F}(1,18)=18.41, \mathrm{p}<0.01$. Baseline $(\mathrm{M}=3.87, \mathrm{SD}=1.56)$, NoteCoStruct $(M=6.23, S D=0.79))$. We also found that learners had higher levels of cognitive engagement $(F(1,18)=4.45, \mathrm{p}<0.05$. Baseline $(M=5.50, S D=0.77)$, NoteCoStruct $(M=6.33, S D=0.98))$ and emotional engagement $(F(1,18)=11.49, \mathrm{p}<0.01$. Baseline $(\mathrm{M}=4.73$, $\mathrm{SD}=0.84)$, NoteCoStruct $(\mathrm{M}=6.10, \mathrm{SD}=0.96))$ while using NoteCoStruct than using the baseline system.

\section{DISCUSSION}

Enhancing online learners' sense of community and making students feel more engaged has long been a major goal of designers of online education. With the nature of remote settings, interacting and sharing information with peers are impeded, which tends to cause feelings of isolation and lack of study motivation [1]. Our prototype enables one-way asynchronous and indirect interactions with peers through note-sharing, and our work investigates whether seeing peers' notes of two different formats (learning traces versus full notes) can have differential effects on learners. Results from our preliminary data analysis showed that learning with NoteCoStruct significantly enhanced learners' sense of community and engagement cognitively and emotionally.

Compared with full notes, learning traces generated by NoteCoStruct learners are in multiple forms (highlights, short comments, and finalized full notes) and can provide richer social contexts to other learners. For example, peers' highlights may inform learners what are the most important parts that they should pay special attention to when they are watching the lecture video, while shared full notes that lack specificity to different parts of the video can barely be helpful in this respect. Thus, learners using NoteCoStruct may not only be academically guided and supported by peers, but also feel their needs in learning from videos being fulfilled, which may have contributed to fostering a sense of fellowship. Although in the baseline system, the amount of information included in full notes may be able to provide similar academic support, the holistic, unsegmented notes can add extra burdens to learners, and learners might be reluctant to read them thoroughly. In contrast, learning traces shown to learners using NoteCoStruct are small-packaged information for providing immediate support to note-taking, thus more likely to be proactively digested by learners. The lower level of distraction associated with NoteCoStruct provides support to this observation.

With the preliminary results, we have shown that NoteCoStruct has the potential to foster a sense of learning community by accumulating and displaying learning traces that scaffold subsequent learners. To further investigate how NoteCoStruct system affects online learners' note-taking behaviors in depth, we plan to conduct content analysis and compare the notes generated by users learning with NoteCoStruct versus the baseline system. We noticed that one limitation of the current system design is that learners using NoteCoStruct spend more time on taking notes than traditional learners, thus, future work can investigate how to mitigate the potential downsides this may cause. Furthermore, future work can look into how different content of learning traces, for example, academic oriented (e.g., notes about understanding of a taught definition) versus emotion oriented (e.g., notes about perceived difficulty of 
the course), may affect subsequent learners' sense of community and learning outcomes.

\section{ACKNOWLEDGMENTS}

The authors would like to thank study participants for their time and effort and anonymous reviewers for their insightful feedback on the paper. The work is supported in part by UC Davis through HaoChuan Wang's startup grant and the Global Research Experience in Advanced Technologies (GREAT) Program at UC Davis.

\section{REFERENCES}

[1] Azad Ali and David Smith. 2015. Comparing social isolation effects on students attrition in online versus face-to-face courses in computer literacy. Issues in Informing Science and Information Technology 12, 1 (2015), 11-20.

[2] Hanna Ashar and Robert Skenes. 1993. Can Tinto's student departure model be applied to nontraditional students? Adult education quarterly 43, 2 (1993), 90-100.

[3] Lori Breslow, David E Pritchard, Jennifer DeBoer, Glenda S Stump, Andrew D Ho, and Daniel T Seaton. 2013. Studying learning in the worldwide classroom research into edX's first MOOC. Research \& Practice in Assessment 8 (2013), $13-25$.

[4] Ruth E Brown. 2001. The process of community-building in distance learning classes. fournal of asynchronous learning networks 5, 2 (2001), 18-35.

[5] Gerald F Burch, Nathan A Heller, Jana J Burch, Rusty Freed, and Steve A Steed. 2015. Student engagement: Developing a conceptual framework and survey instrument. Fournal of Education for Business 90, 4 (2015), 224-229.

[6] Robert M Carini, George D Kuh, and Stephen P Klein. 2006. Student engagement and student learning: Testing the linkages. Research in higher education 47, 1 (2006), 1-32.

[7] L Brent Igo, Roger Bruning, and Matthew T McCrudden. 2005. Exploring Differ ences in Students' Copy-and-Paste Decision Making and Processing: A MixedMethods Study. Fournal of Educational Psychology 97, 1 (2005), 103.

[8] Geoff Isaacs. 1994. Lecturing practices and note-taking purposes. Studies in Higher Education 19, 2 (1994), 203-216.

[9] Genevieve Marie Johnson. 2006. Synchronous and asynchronous text-based CMC in educational contexts: A review of recent research. TechTrends 50, 4 (2006) 46-53.

[10] Richard D Johnson, Steven Hornik, and Eduardo Salas. 2008. An empirical examination of factors contributing to the creation of successful e-learning environments. International fournal of Human-Computer Studies 66, 5 (2008), 356-369.
[11] Kenneth A Kiewra. 1989. A review of note-taking: The encoding-storage paradigm and beyond. Educational Psychology Review 1, 2 (1989), 147-172.

[12] Melina Klepsch, Florian Schmitz, and Tina Seufert. 2017. Development and validation of two instruments measuring intrinsic, extraneous, and germane cognitive load. Frontiers in psychology 8 (2017), 1997.

[13] Chinmay Kulkarni and Ed Chi. 2013. All the news that's fit to read: a study of social annotations for news reading. In Proceedings of the SIGCHI Conference on Human Factors in Computing Systems. Association for Computing Machinery, New York, NY, USA, 2407-2416.

[14] Yi-Chieh Lee, Wen-Chieh Lin, Fu-Yin Cherng, Hao-Chuan Wang, Ching-Ying Sung, and Jung-Tai King. 2015. Using time-anchored peer comments to enhance social interaction in online educational videos. In Proceedings of the 33rd Annual ACM Conference on Human Factors in Computing Systems. Association for Computing Machinery, New York, NY, USA, 689-698.

[15] Ching Liu, Chi-Lan Yang, Joseph Jay Williams, and Hao-Chuan Wang. 2019. NoteStruct: Scaffolding Note-taking while Learning from Online Videos. In Extended Abstracts of the 2019 CHI Conference on Human Factors in Computing Systems. Association for Computing Machinery, New York, NY, USA, 1-6.

[16] Maslawati Mohamad and Shahizan Shaharuddin. 2014. Online Forum Discussion to Promote Sense of Learning Community among the Group Members. International Education Studies 7, 13 (2014), 61-74.

[17] Pam A Mueller and Daniel M Oppenheimer. 2014. The pen is mightier than the keyboard: Advantages of longhand over laptop note taking. Psychological science 25, 6 (2014), 1159-1168.

[18] Elena Novak, Rim Razzouk, and Tristan E Johnson. 2012. The educational use of social annotation tools in higher education: A literature review. The Internet and Higher Education 15, 1 (2012), 39-49.

[19] Janne Pietarinen, Tiina Soini, and Kirsi Pyhältö. 2014. Students' emotional and cognitive engagement as the determinants of well-being and achievement in school. International fournal of Educational Research 67 (2014), 40-51.

[20] Tae Eun Shim and Song Yi Lee. 2020. College students' experience of emergency remote teaching due to COVID-19. Children and youth services review 119 (2020), 105578.

[21] Na Sun, Mary Beth Rosson, and John M Carroll. 2018. Where is community among online learners? Identity, efficacy and personal ties. In Proceedings of the 2018 chi conference on human factors in computing systems. Association for Computing Machinery, New York, NY, USA, 1-13.

[22] Peggy Van Meter, Linda Yokoi, and Michael Pressley. 1994. College students' theory of note-taking derived from their perceptions of note-taking. Fournal of Educational Psychology 86, 3 (1994), 323.

[23] Claire E Weinstein and Richard E Mayer. 1983. The teaching of learning strategies. Innovation abstracts 5, 32 (1983), 315-327.

[24] Suzanne Young and Mary Alice Bruce. 2011. Classroom community and student engagement in online courses. Journal of Online Learning and Teaching 7, 2 (2011), 219-230. 